\title{
On-Off-On Fluorescent Chemosensors Based on N/P-Codoped Carbon Dots for Detection of Microcystin-LR
}

Xiaoyan Wang ${ }^{\dagger, *}$, Jialuo $\mathrm{Yu}^{\ddagger}, *$, Wan $\mathrm{Ji}^{\dagger}$, Maryam Arabi ${ }^{\ddagger}, *$, Longwen $\mathrm{Fu}^{\ddagger}, *$, Bowei $\mathrm{Li}^{\ddagger, *}$, Lingxin

Chen $^{\dagger, \ddagger, \#, *}$

${ }^{\dagger}$ School of Pharmacy, Binzhou Medical University, Yantai 264003, China

$\$$ CAS Key Laboratory of Coastal Environmental Processes and Ecological Remediation, Shandong Key Laboratory of Coastal Environmental Processes, Research Centre for Coastal Environmental Engineering and Technology, Yantai Institute of Coastal Zone Research, Chinese Academy of Sciences, Yantai 264003, China

"Center for Ocean Mega-Science, Chinese Academy of Sciences, Qingdao 266071, China

*Corresponding authors.

E-mail addresses: wangxy@yic.ac.cn (X Wang); 1xchen@yic.ac.cn (L Chen) 


\section{Contents}

Materials. Microcystin-LR (MC-LR) and microcystin-RR (MC-RR) were purchased from Algalchem Inc. (Taiwan, China). Adenosine 5'-triphosphate disodium salt hydrate (ATP) was obtained from Sangon Biotech Co. (Shanghai, China). Acetamiprid, L-threonine, L-alanine, L-arginine, L-leucine, and L-histidine were supplied by Aladdin Co. (Shanghai, China). Niclosamide and dinotefuran were purchased from Macklin Co. (Shanghai, China). Metal salts, including $\mathrm{NiCl}_{2}, \mathrm{~Pb}\left(\mathrm{NO}_{3}\right)_{2}, \mathrm{NaCl}, \mathrm{HgCl}_{2}, \mathrm{MgCl}_{2}, \mathrm{CuCl}_{2}$, and $\mathrm{FeCl}_{3}$, were obtained from Sinopharm Chemical Reagent Co. (Shanghai, China). Deionized water with the specific resistance of $18.2 \mathrm{M} \Omega$ was produced from a Milli-Q gradient water purification system (Millipore, USA).

Instrumentations. Fluorescence measurements were performed by a Fluoromax-4 Spectrofluorometer (Horiba Scientific), and ultraviolet-visible (UV-vis) spectra were measured on a Thermo Scientific NanoDrop 2000/2000C spectrophotometer (Thermo Fisher Scientific, Waltham, MA). The fluorescence time scan was recorded by an F-4700 FL spectrophotometer (Hitachi), and the QY was obtained by an Absolute PL quantum yield spectrometer (C9920-02G, HAMAMATSU). Fluorescence lifetimes were measured on an Edinburgh Instruments FLS1000 modular fluorescence and phosphorescence spectrometer. The zeta potential was examined by a Malvern Zetasizer Nano-ZS90 (ZEN3590, UK). The morphologies of the materials were characterized by transmission electron microscopy (TEM, JEM-1230, operating at $100 \mathrm{kV}$ ). Energy-dispersive X-ray spectroscopy (EDXS) data were acquired by a scanning electron microscopy (SEM, Hitachi S-4800 FE-SEM, operating at $5 \mathrm{kV}$ ) system equipped with an EDAX-PHOE-NIX energy spectrum probe. $\mathrm{X}$-ray photoelectron spectroscopy (XPS) measurements were taken on an ESCALAB $\mathrm{Xi+}$ spectrometer (Thermo Scientific, USA). Fourier transform infrared (FT-IR) spectroscopy (Nicolet iS10, Thermo Fisher) was used for the identification of functional groups.

Synthesis of N/P CDs. First, $1 \mathrm{~g}$ of ATP was dispersed in $30 \mathrm{~mL}$ of ultrapure water to obtain a clear suspension, which was transferred into a $50 \mathrm{~mL}$ Teflon-lined autoclave and heated at $220{ }^{\circ} \mathrm{C}$ for $6 \mathrm{~h}$. The obtained CD solution (concentration of $32.3 \mathrm{~g} / \mathrm{L}$ ) was stored at $4{ }^{\circ} \mathrm{C}$ in the dark before use.

HPLC-MS determination. The concentrations of MC-LR in the spiked real water samples were determined by using conventional techniques and HPLC-MS (TSQ Quantum Access M, Thermo Fisher). A reversed-phase $\mathrm{C}_{18}$ column $(150 \mathrm{~mm} \times 4.6 \mathrm{~mm}$, i.d. $3.5 \mu \mathrm{m}$, Thermo Scientific $)$ was used for LC separation. The column oven temperature was $30{ }^{\circ} \mathrm{C}$, and the injection volume was $10 \mu \mathrm{L}$. The flow rate was $0.3 \mathrm{~mL} / \mathrm{min}$ with a gradient mode of $\mathrm{t}=0 \min (20: 80), \mathrm{t}=3 \min (60: 40), \mathrm{t}$ $=4 \min (60: 40), t=6 \min (80: 20), t=10 \min (20: 80)$ and $\mathrm{t}=20 \min (20: 80)$ for acetonitrile and $0.1 \%$ 
formic acid, respectively. The mass spectrometer was operated in positive ion mode with full scans, a spray voltage of $3.5 \mathrm{kV}$, and capillary temperature of $270{ }^{\circ} \mathrm{C}$.

A

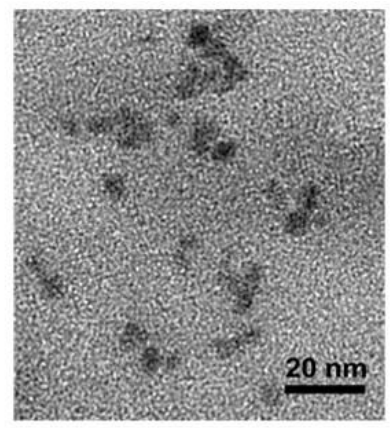

B

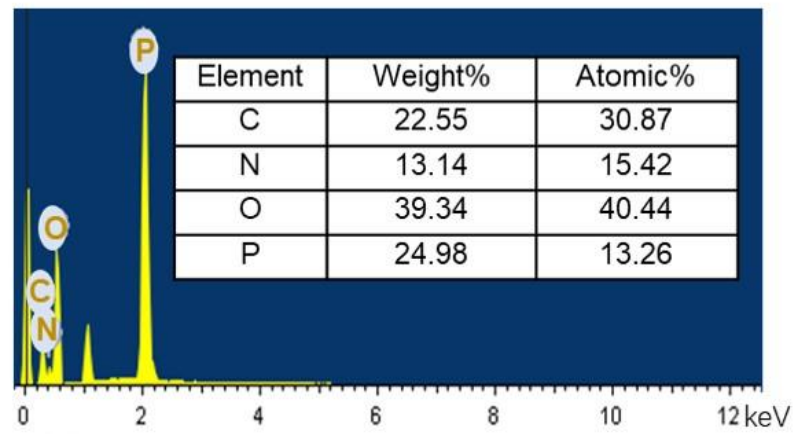

Figure S1. (A) TEM image, (B) EDXS spectrum and weight percentage of elements (inset Table) of the as-prepared N/P CDs.
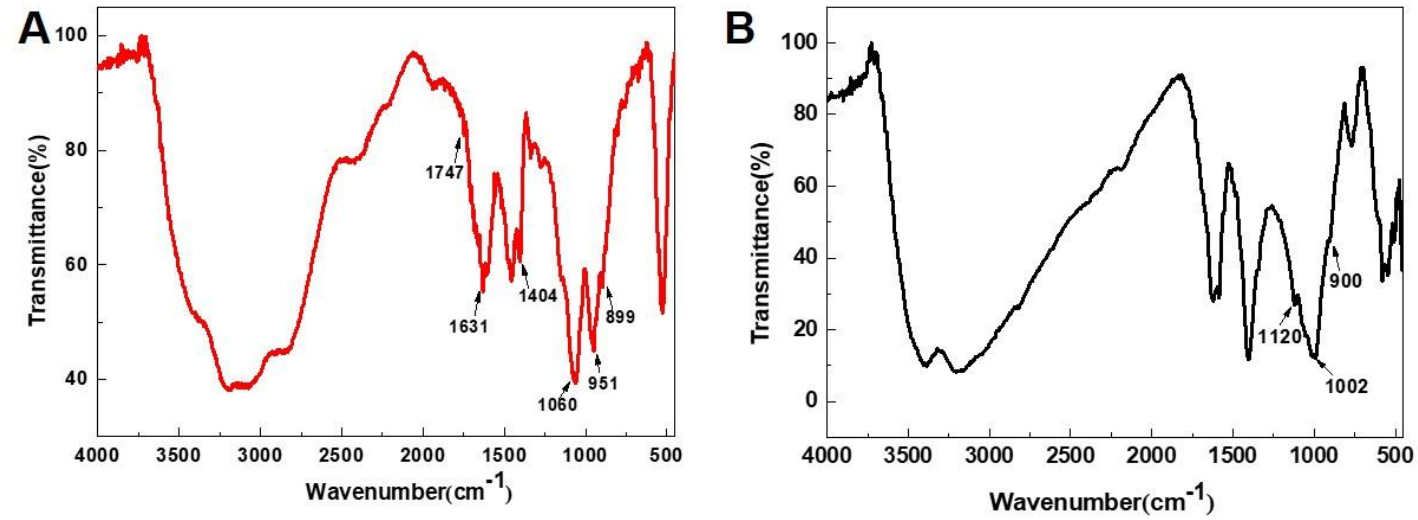

Figure S2. FT-IR spectra of N/P CDs before (A) and after (B) the addition of $\mathrm{Fe}^{3+}$. 


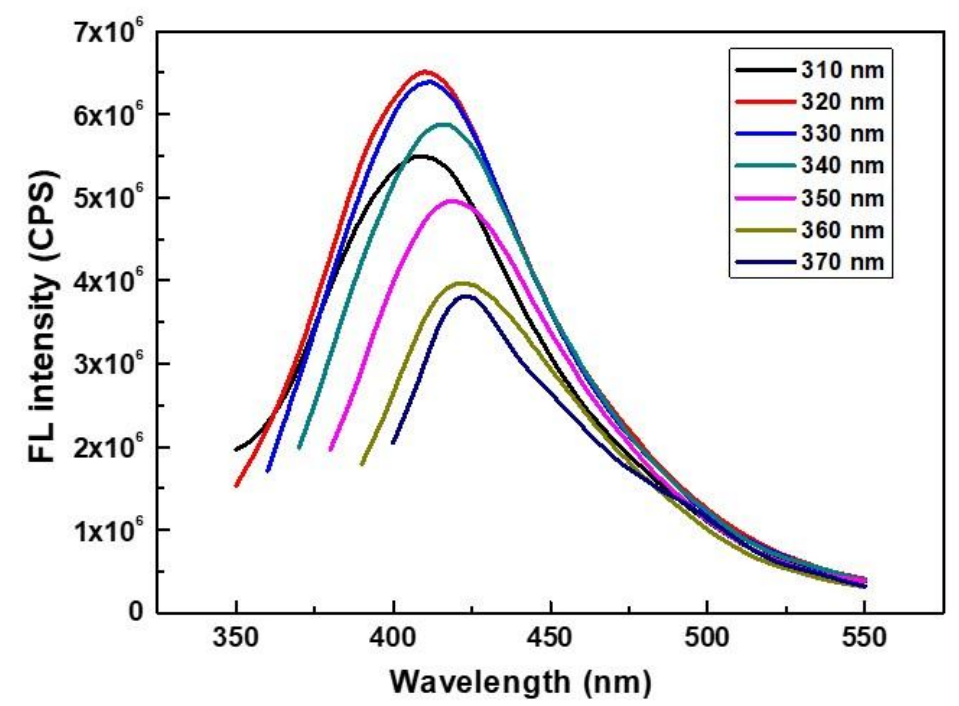

Figure S3. Fluorescence emission spectra of N/P CDs changing with various excitation wavelengths.
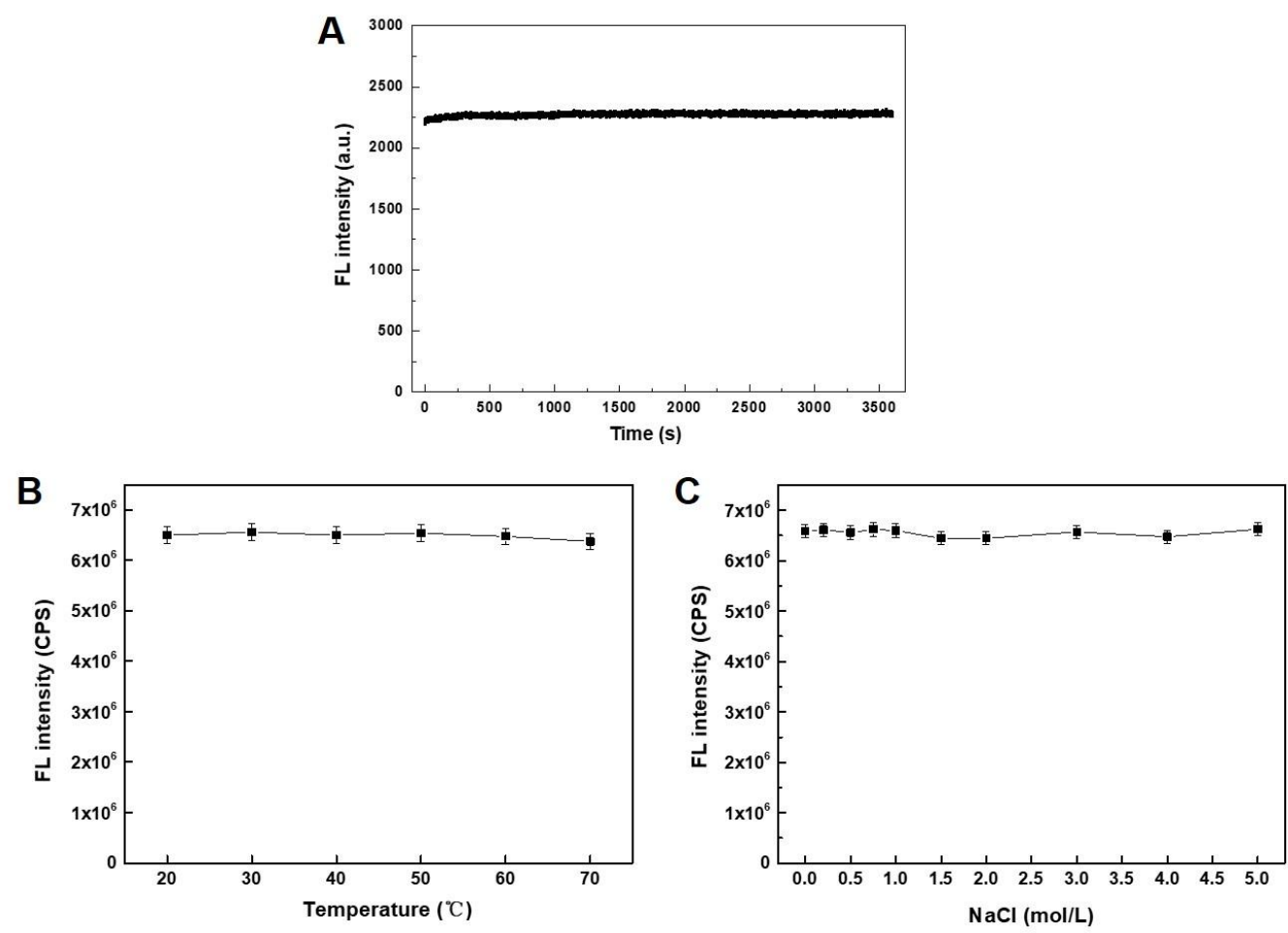

Figure S4. (A) Fluorescence intensity changes of the N/P CDs for $1 \mathrm{~h}$ under $320 \mathrm{~nm}$ excitation with data collection once every second. The effect of temperature (B) and ionic strengths (C) on the fluorescence intensity of the N/P CDs. 


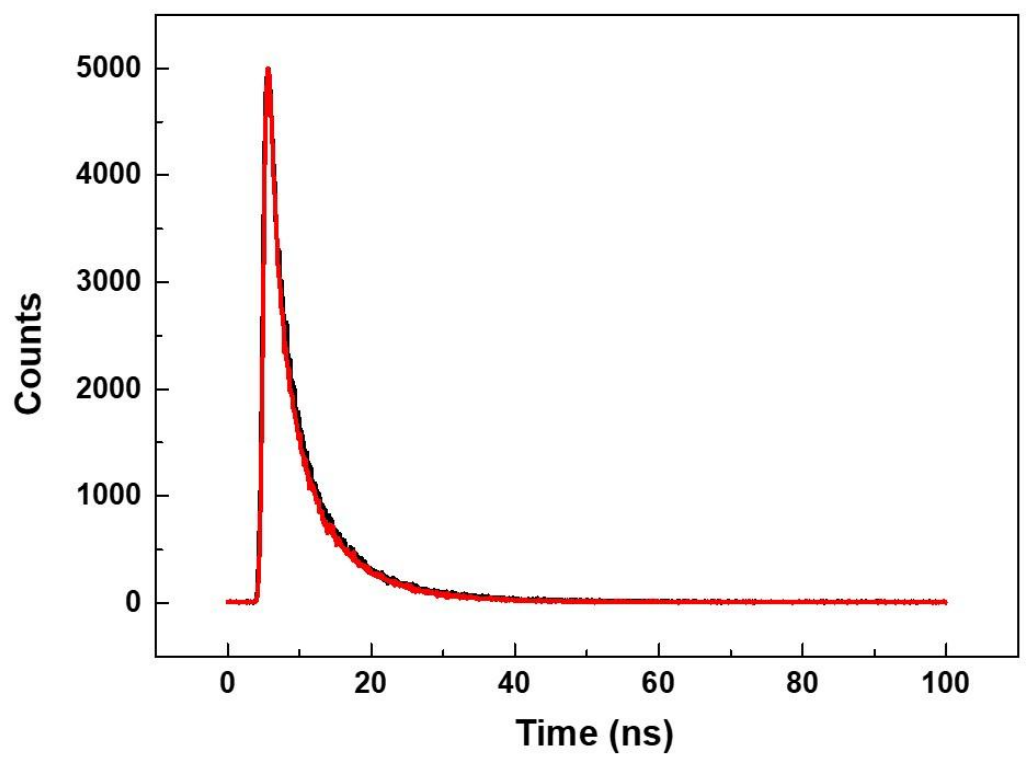

Figure S5. The fluorescence lifetime of the N/P CDs in the absence (red line) and presence (black line) of $\mathrm{Fe}^{3+}$.
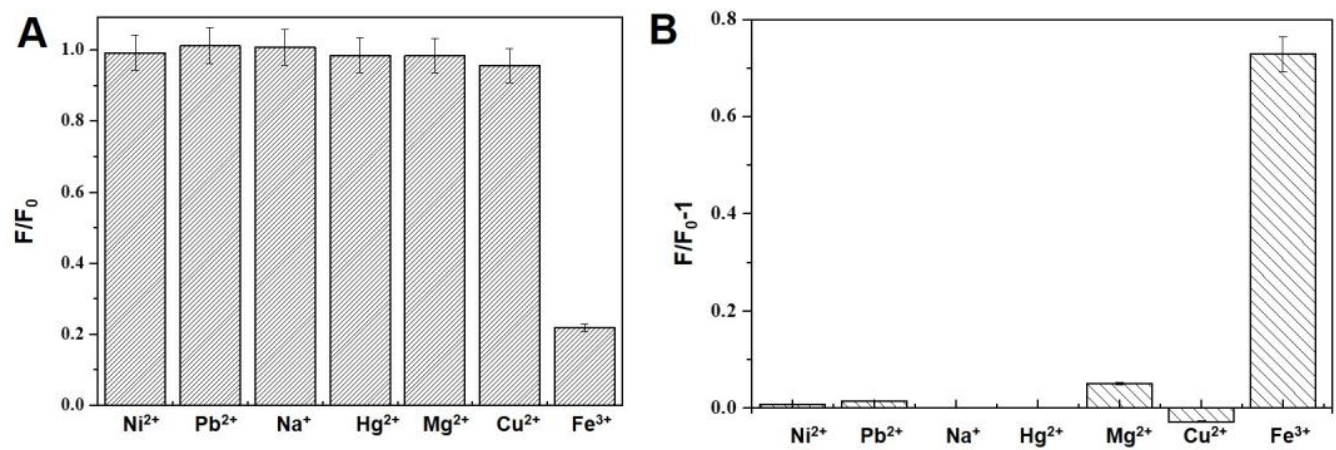

Figure S6. (A) Effect of various metal ions $\left(\mathrm{Ni}^{2+}, \mathrm{Pb}^{2+}, \mathrm{Na}^{+}, \mathrm{Hg}^{2+}, \mathrm{Mg}^{2+}, \mathrm{Cu}^{2+}\right.$ and $\left.\mathrm{Fe}^{3+}\right)$ at a concentration of $0.5 \mathrm{mmol} / \mathrm{L}$ on the fluorescence intensity of $\mathrm{N} / \mathrm{P} \mathrm{CD}$ aqueous solution (the concentration of N/P CDs was $5.38 \mathrm{mg} / \mathrm{L}$ ); (B) Fluorescence recovery effects of N/P CD-metal ions in the presence of MC-LR at a concentration of $1 \mu \mathrm{g} / \mathrm{L}$ under excitation at $320 \mathrm{~nm}$. 

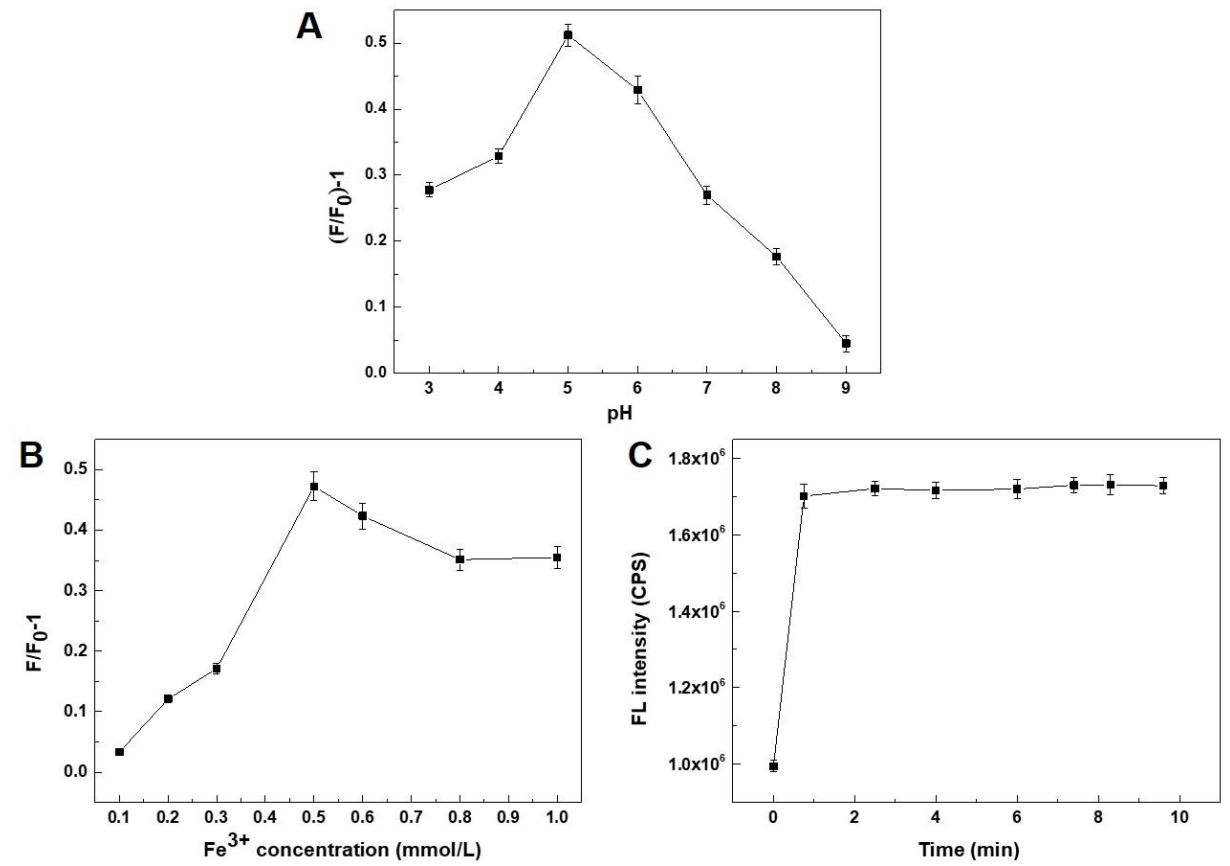

Figure S7. (A) Effect of $\mathrm{pH}$ on the fluorescence intensity of the sensor in the presence of $1 \mu \mathrm{g} / \mathrm{L}$ MC-LR. (B) The influence of different concentrations of $\mathrm{Fe}^{3+}$ on the fluorescence intensity recovery of the N/P CD-Fe ${ }^{3+}$ system (the concentrations of the N/P CDs and MC-LR were $5.38 \mathrm{mg} / \mathrm{L}$ and 0.5 $\mu \mathrm{g} / \mathrm{L}$, respectively). (C) Fluorescence response time of the sensor for MC-LR detection. The fluorescence intensity was recorded at a wavelength of $420 \mathrm{~nm}$.
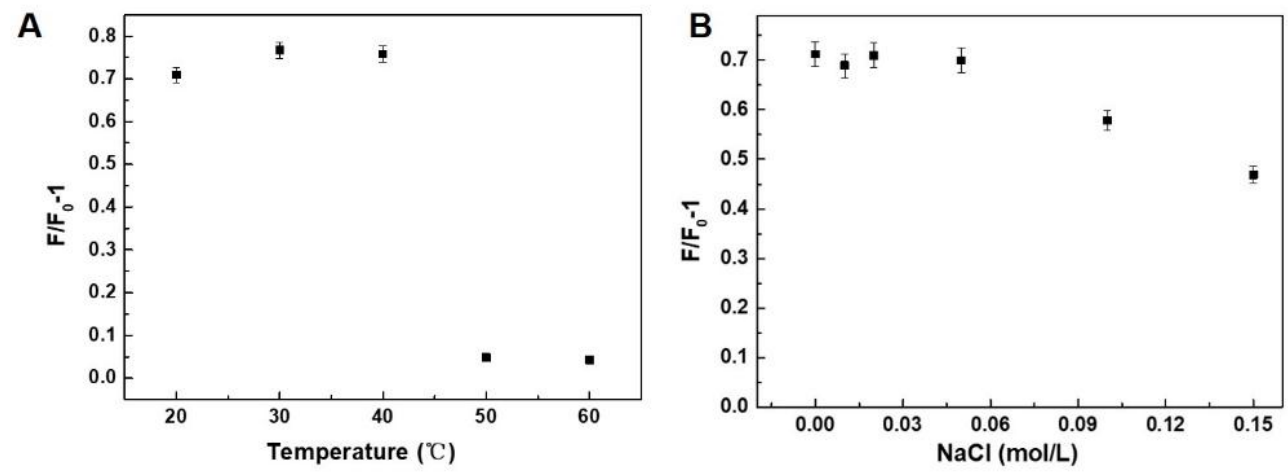

Figure S8. Effect of temperature (A) and ionic strength (B) on the fluorescence intensity of chemosensors in the presence of $1 \mu \mathrm{g} / \mathrm{L}$ MC-LR. 